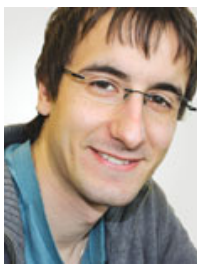

Marc Erhardt

Jahrgang 1981. 2002-2006 Biologiestudium, Universitäten UIm und Konstanz. 2006 Diplomarbeit an der University of Utah, Salt Lake City, USA. 2007-2011 Promotion bei Prof. Dr. K. T. Hughes, University of Utah, und Prof. Dr. W. Boos, Universität Konstanz, Stipendium Boehringer Ingelheim Fonds. 2011-2012 Marie-Curie-Postdoc und Gruppenleiter an der Université de Fribourg, Schweiz. Ab 2013 Helmholtz-Nachwuchsgruppenleiter am HelmholtzZentrum für Infektionsforschung in Braunschweig.

DOI: $10.1007 / \mathrm{s} 12268-012-0245-1$

(c) Springer-Verlag 2012

Flagellen sind einzigartige Maschinen im Nanomaßstab, durch deren Rotation sich Bakterien in flüssiger Umgebung fortbewegen. Die Fähigkeit, sich fortzubewegen, ist von groBer Wichtigkeit für die Virulenz des Gramnegativen Pathogens Salmonella enterica. Strukturell besteht das bakterielle Flagellum von Salmonella aus drei Hauptbestandteilen: (1) ein Basalkörper, welcher beide Zellmembranen durchspannt und einen flagellumspezifischen Typ-III-Sekretionsapparat beinhaltet; (2) ein flexibles, universelles Gelenkstück und (3) ein starres, externes Filament. Das Typ-III-Sekretionssystem ist verantwortlich für den Export eines Großteils der strukturellen Bestandteile dieser Nanomaschine und vereint viele funktionelle Gemeinsamkeiten mit dem Virulenz-assoziierten Typ-IIIInjektisomkomplex. Der Membran-Protonengradient stellt die Energie für den Proteinexport durch diesen Typ-III-Sekretionsapparat bereit und nicht, wie ursprünglich vermutet,

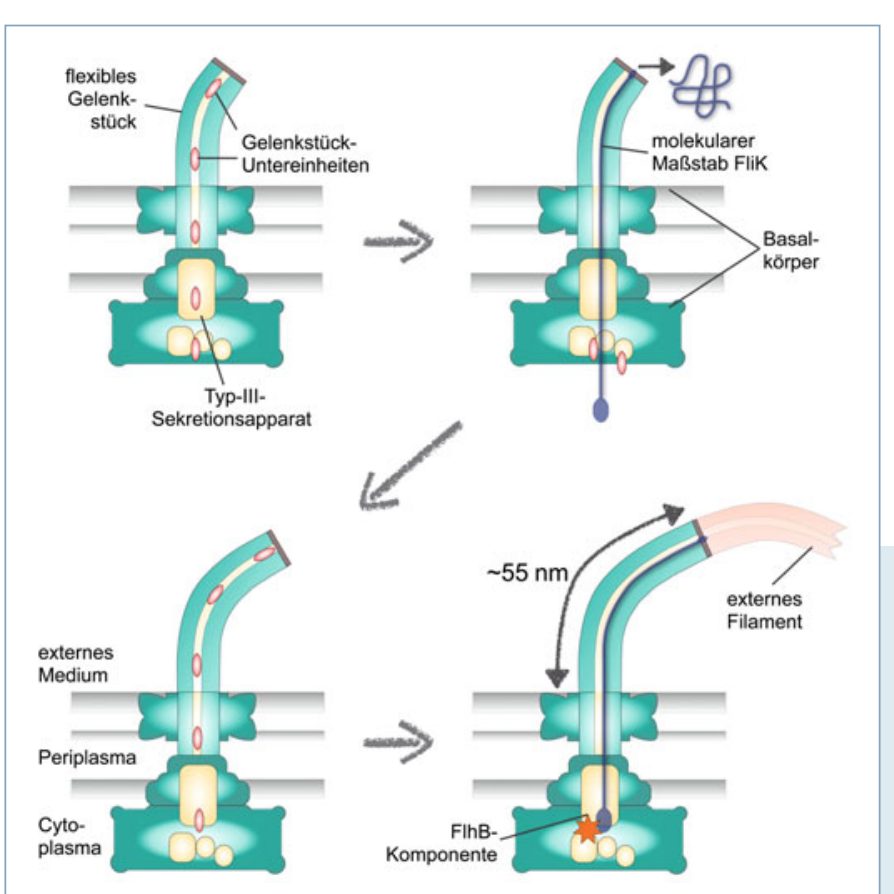

MARC ERHARDT

Elisabeth-Gateff-Preis 2012 der GfG

\title{
Biosynthese des bakteriellen Flagellums - in vivo-Längenmessung im Nanomaßstab
}

DÉPARTEMENT DE MÉDECINE, UNIVERSITÉ DE FRIBOURG, SCHWEIZ

die Hydrolyse von ATP, da auch Deletionsmutanten der flagellumspezifischen ATPase FliI zur Fortbewegung fähig sind [1]. Neben der ATPase FliI sind auch weitere Untereinheiten des Transportsystems, wie der zytoplasmatische C-Ring, nicht für den eigentlichen Transportprozess nötig, sondern erfüllen eine unterstützende Funktion [2].

Die Länge von Substrukturen des bakteriellen Flagellums wird durch spezifische Mechanismen genau kontrolliert. Besonders die Länge des flexiblen Gelenkstücks, welches den Basalkörper des Flagellums mit dem starren Filament außerhalb der Zelle verbindet, ist in Salmonella enterica auf 55 plus/minus sechs Nanometer festgelegt. Die grundlegende Frage war für lange Zeit, wie eine bakterielle Zelle die Länge dieser externen Struktur messen und diese Längeninformation verarbeiten kann. Dieser Prozess wird in Salmonella durch die Sekretion eines molekularen Maßbands ermöglicht (Abb. 1). Das Maßbandprotein FliK wird konstant, aber abwechselnd mit Untereinheiten des Gelenkstücks während dessen Aufbaus sekretiert. FliK misst hierbei die Länge des Gelenkstücks und überträgt diese Information an die FlhBKomponente des TypIII-Sekretionssystems in der zytoplasmatischen Membran. Eine Interaktion zwischen dem Carboxylende von FliK und FlhB induziert einen Wechsel in der Erken- nungsspezifität des Typ-III-Sekretionssystems. Dieser Wechsel führt dazu, dass anstatt Komponenten des Basalkörpers nun die Untereinheiten des Filaments exportiert werden [3, 4].

Die Fähigkeit zur Fortbewegung und der Proteinexport über Typ-III-Sekretionssysteme sind essenziell für die Pathogenität vieler Bakterien. In Anbetracht der fortschreitenden Entwicklung von Resistenzen gegen vorhandene Antibiotika, ist die Entwicklung neuer Wirkstoffe gegen spezifische Mechanismen pathogener Bakterien ein wichtiges Ziel der mikrobiellen Forschung. Ein Verständnis der grundlegenden molekularen Abläufe ist hierbei von entscheidender Bedeutung und könnte neue Ansatzpunkte für antimikrobielle Therapien liefern.

\section{Literatur}

[1] Paul K, Erhardt M, Hirano T et al. (2008) Energy source of flagellar type III secretion. Nature 451:489-492

[2] Erhardt M, Hughes KT (2010) C-ring requirement in flagellar type III secretion is bypassed by FlhDC upregulation. Mol Microbiol 75:376-393

[3] Erhardt M, Hirano T, Yichu S et al. (2010) The role of the FliK molecular ruler in hook-length control in Salmonella enterica. Mol Microbiol 75:1272-1284

[4] Erhardt M, Singer HM, Wee DH et al. (2011) An infrequent molecular ruler controls flagellar hook length in Salmonella enterica. EMBO J 30:2948-2961

\section{Korrespondenzadresse: \\ Dr. Marc Erhardt \\ Université de Fribourg \\ Départment of Medicine \\ Chemin du Musée 10 \\ $\mathrm{CH}-1700$ Fribourg \\ Tel.: +41-(0)26-300-9435 \\ marc.erhardt@unifr.ch}

Abb. 1: Der molekulare Maßstab, das Protein Flik, und Untereinheiten des Gelenkstücks werden abwechselnd während der Biosynthese des Flagellums exportiert. Während einer Längenmessung durch den molekularen Maßstab pausiert temporär der Export von Gelenkstück-Untereinheiten. Nach der Messung wird FliK in die Umgebung sekretiert. In Gelenkstücken mit der physiologischen Länge von 55 Nanometern findet eine produktive Interaktion des Maßstabs mit der FlhB-Komponente des Typ-III-Sekretionssystems statt, und dies induziert einen Wechsel in der Erkennungsspezifität des Exportapparats (roter Stern). 